\title{
Ground-state Wigner functional of linearized gravitational field
}

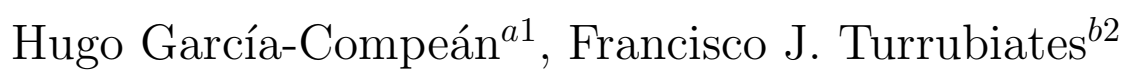 \\ ${ }^{a}$ Departamento de Física, Centro de Investigación y de Estudios Avanzados del IPN \\ P.O. Box 14-740, 07000 México D.F., México. \\ ${ }^{b}$ Departamento de Física, Escuela Superior de Física y Matemáticas del IPN \\ Unidad Adolfo López Mateos, Edificio 9, 07738, México D.F., México.
}

\begin{abstract}
The deformation quantization formalism is applied to the linearized gravitational field. Standard aspects of this formalism are worked out before the ground state Wigner functional is obtained. Finally, the propagator for the graviton is also discussed within the context of this formalism.
\end{abstract}

September 5, 2011

\footnotetext{
${ }^{1}$ e-mail address: compean@fis.cinvestav.mx

${ }^{2}$ e-mail address: fturrub@esfm.ipn.mx
} 


\section{Introduction}

The study of weak gravitational fields has produced important results during the years. One of them is the prediction of gravitational waves for the non-stationary cases. This has been a very important area of research and many groups around the world are working on its detection through bars and interferometers (for a review involving the the experimental and theoretical parts, see for instance, [1, 2, 3]). It is expected that they will be a reality within the next few years.

From the theoretical point of view ADM's formalism [4, 5] allowed for the first time to deal the gravitational field from a suitable point of view to study its mathematical structure. In particular the weak field was also incorporated into this framework. In the present case we are not considering the study of sources by themselves and all the analysis is performed in the vacuum. Many questions can also be treated for this case just as finding the corresponding ground state of the field, that is the vacuum. Some remarkable results dealing with this problem had been obtained previously by Kuchar [6] and Hartle [7]. In these works the canonical quantization and path integral procedures were employed to obtain the ground state wave function of linearized gravity. However there exists another method of quantization the so called deformation quantization formalism [8] that can be also applied to the gravitational field [9, 10]. In a recent work we employed such technique to deal with some cosmological models in the minisuperspace [11]. The present paper is motivated by the possibility to extend some of the results that we obtained there to some other interesting cases in general relativity and its approaches.

The ideas of deformation quantization are based on the fundamental works of Weyl, Wigner, Groenewald, Moyal, Gerstenhaber and Vey (for some recent reviews and developments on the subject, see for instance, [12, 13]) that evolved during the years until the introduction of the theory in its final form given by Bayen et al [8]. This quantization comes from a deformation of the usual algebra product of smooth functions on the classical phase space which then induce a deformation of the Poisson bracket algebra. It has been proved that this new product called the $\star$-product exists for any symplectic or Poisson manifold [14, 15]. In terms of this quantization it is not necessary the use of operators or even the introduction a Hilbert space, which in principle offers important advantages when we deal with systems whose phase space has non-trivial topology [14, 15]. For these reasons we consider that this method is suitable to deal with certain problems at quantum level of the gravitational field in the phase space.

The main goal of the present paper is to obtain by this procedure the ground state Wigner functional of linearized gravity and compare it with the previous results

[6, 7. At the same time we look for to present a new example of the application of the deformation quantization formalism, in particular to field theory, since there are just few of them. Finally, we also hope that the results presented here will motivate the application of this method of quantization to different configurations and problems involving the gravitational field.

Our paper is organized as follows. In section 2 we introduce the conventions and notation of the linearized gravitational field required to apply the deformation quantization formalism. The deformation quantization of the linearized gravitational field 
is performed in the temporal gauge and in the (TT)-gauge in section 3. In particular, in this same section, the Wigner functional for the ground state, the normal ordering operator and the graviton propagator are obtained. Finally in section 4 we give our conclusions and final remarks.

\section{Overview of the Linearized Gravitational Field}

We start by considering the Fierz-Pauli action defined in a Minkowski spacetime with signature $(-,+,+,+)$

$$
\mathcal{L}=-\frac{1}{4} h_{\alpha \beta, \lambda} h_{, \lambda}^{\alpha \beta}-\frac{1}{2} h_{\alpha, \lambda}^{\alpha} h_{, \beta}^{\lambda \beta}+\frac{1}{4} h_{\alpha, \lambda}^{\alpha} h_{\beta}^{\beta, \lambda}+\frac{1}{2} h_{\beta, \alpha}^{\alpha} h_{, \lambda}^{\beta \lambda},
$$

where $\alpha, \beta$, etc. $=0,1,2,3$ and $i, j$, etc. $=1,2,3$. Along the paper it is assumed the Einstein's convention on the sum over the repeated indices. Moreover we have used the notation $h_{\alpha \beta, \lambda}=\nabla_{\lambda} h_{\alpha \beta}$. The fields $h_{\alpha \beta}(\vec{x}, t)$ are dynamical, in particular for a $(3+1)$-decomposition of spacetime, $h_{00}, h_{0 i}$ and $h_{i j}$ are the relevant variables. The canonical momenta corresponding to these fields are

$$
\pi_{00}=\frac{\delta L}{\delta \dot{h}_{00}}, \quad \pi_{0 i}=\frac{\delta L}{\delta \dot{h}_{0 i}}, \quad \pi_{i j}=\frac{\delta L}{\delta \dot{h}_{i j}},
$$

where $\dot{h}_{i j}=h_{i j, 0}=\frac{\partial h_{i j}}{\partial x^{0}}$ is the trace of $h_{i j}$.

They can be computed from the action (11) and they take the following explicit form

$$
\pi_{00}=\frac{1}{2} h_{0 k, k}, \quad \pi_{0 i}=\frac{1}{2} h_{l l, k}-\frac{1}{2} h_{00, k}-h_{l k, l}, \quad \pi_{i j}=\frac{1}{2}\left[\dot{h}_{i j}+\delta_{i j}\left(-\dot{h}_{k k}+h_{k, k}\right)\right],
$$

where $h_{k k}=\operatorname{Tr}\left(h_{i j}\right)$.

The corresponding Hamiltonian can be written as

$$
\begin{aligned}
H & =\int d^{3} x \mathcal{H} \\
& =\int d^{3} x\left(\pi_{00} \dot{h}_{00}+\pi_{0 k} \dot{h}_{0 k}+\pi_{i j} \dot{h}_{i j}\right)-L,
\end{aligned}
$$

where $L=\int d^{4} x \mathcal{L}$ and the hamiltonian density reads

$$
\begin{aligned}
\mathcal{H} & =\pi_{i j} \pi_{i j}-\frac{1}{2} \pi_{m m} \pi_{n n}+\frac{1}{2} \pi_{m m} h_{0 k, k}+\frac{1}{8} h_{0 k, k} h_{0 l, l}+\frac{1}{2} h_{00, m} h_{l l, m}-\frac{1}{4} h_{l l, m} h_{k k, m} \\
& -\frac{1}{2} h_{0 k, m} h_{0 k, m}+\frac{1}{4} h_{k l, m} h_{k l, m}-\frac{1}{2} h_{m l, m} h_{n l, n}-\frac{1}{2} h_{00, n} h_{m n, m}+\frac{1}{2} h_{m n, m} h_{l l, n} .
\end{aligned}
$$

In the present paper we are going to work in the temporal gauge where $h_{\alpha 0}=0$. In this gauge we have that the Poisson bracket (at the same fixed time) between $h_{i j}(\vec{x}, t)$ and $\pi^{k l}(\vec{x}, t)$ is given by

$$
\left\{h_{i j}(\vec{x}, t), \pi^{k l}\left(\vec{x}^{\prime}, t\right)\right\}_{P B}=\delta_{i j}^{k l} \delta\left(\vec{x}-\vec{x}^{\prime}\right),
$$


where $\delta_{i j}^{k l}=\frac{1}{2}\left(\delta_{i}^{k} \delta_{j}^{l}+\delta_{j}^{k} \delta_{i}^{l}\right)$. The rest of Poisson brackets vanish

$$
\left\{h_{i j}(\vec{x}, t), h_{k l}\left(\vec{x}^{\prime}, t\right)\right\}_{P B}=0=\left\{\pi^{i j}(\vec{x}, t), \pi^{k l}\left(\vec{x}^{\prime}, t\right)\right\}_{P B} .
$$

The standard expansion of the field variables in the temporal gauge reads

$$
h_{i j}(\vec{x}, t)=\int \frac{d^{3} k}{(2 \pi)^{3 / 2}}\left(\frac{\hbar}{2 \omega(\vec{k})}\right)^{1 / 2}\left(\mathfrak{h}_{i j}(\vec{k}, t) \exp (i \vec{k} \cdot \vec{x})+\mathfrak{h}_{i j}^{*}(\vec{k}, t) \exp (-i \vec{k} \cdot \vec{x})\right)
$$

where $\omega(\vec{k})=|\vec{k}|, \mathfrak{h}_{i j}(\vec{k}, t)=\mathfrak{h}_{i j}(\vec{k}) \exp \{-i \omega(\vec{k}) t\}$ and $\vec{k} \cdot \vec{x} \equiv k_{j} x_{j}$. The momentum is given by

$$
\pi^{i j}(\vec{x}, t)=\int \frac{d^{3} k}{(2 \pi)^{3 / 2}} \sqrt{\frac{\hbar}{2 \omega(\vec{k})}}\left(\mathfrak{p}^{i j}(\vec{k}, t) \exp (i \vec{k} \cdot \vec{x})+\mathfrak{p}^{* i j}(\vec{k}, t) \exp (-i \vec{k} \cdot \vec{x})\right)
$$

where $\mathfrak{p}^{i j}(\vec{k}, t)=\mathfrak{p}^{i j}(\vec{k}) \exp \{-i \omega(\vec{k}) t\}$. From Eq. (3) one can find

$\mathfrak{p}_{i j}(\vec{k}, t)=-\frac{i \omega}{2} \mathfrak{h}_{i j}(\vec{k}, t)+\frac{i \omega}{4} \delta_{i j} \mathfrak{h}_{k k}(\vec{k}, t)+\frac{i}{2}\left[\delta_{i j} k_{k} \mathfrak{h}_{0 k}(\vec{k}, t)-\left(k_{j} \mathfrak{h}_{0 i}(\vec{k}, t)+k_{i} \mathfrak{h}_{0 j}(\vec{k}, t)\right)\right]$.

We will work with the decomposition into the transverse-traceless part $(T T)$, the transverse part $(T)$ and the longitudinal part $(L)$ of this field

$$
h_{i j}(\vec{x}, t)=h_{i j}^{(T T)}(\vec{x}, t)+h_{i j}^{(T)}(\vec{x}, t)+h_{i j}^{(L)}(\vec{x}, t) .
$$

Here the $(T T)$ part satisfies the following conditions:

$$
h_{\alpha 0}^{(T T)}=0, \quad h_{i}^{i(T T)}=0, \quad h_{i j, k}^{(T T)}=0,
$$

which is called the (TT)-gauge.

It is well known that in order to work out with this $(T T)$-gauge is more appropriate (but equivalent) to solve the Gauss law constraint

$$
\nabla_{j} \pi^{i j}(\vec{x})=0 .
$$

Now the decomposition (11) can be introduced through the introduction of a linear projection operator

$$
P_{j k}=\delta_{j k}-\frac{k_{j} k_{k}}{|\vec{k}|^{2}}
$$

with the following properties:

$$
P_{j l} P_{l k}=P_{j k}, \quad P_{j l} k_{l}=0, \quad P_{j j}=2 .
$$

The transverse traceless $(T T)$, transverse $(T)$ and longitudinal $(L)$ parts can be written in terms of the projection operator as

$$
\mathfrak{h}_{j k}^{(T T)}(\vec{k}, t)=P_{j l} P_{m k} \mathfrak{h}_{l m}(\vec{k}, t)-\frac{1}{2} P_{j k}\left(P_{l m} \mathfrak{h}_{l m}(\vec{k}, t)\right),
$$




$$
\begin{gathered}
\mathfrak{h}_{j k}^{(T)}(\vec{k}, t)=\frac{1}{2} P_{j k}\left(P_{l m} \mathfrak{h}_{l m}(\vec{k}, t)\right), \\
\mathfrak{h}_{j k}^{(L)}(\vec{k}, t)=\mathfrak{h}_{j k}(\vec{k}, t)-P_{j l} P_{m k} \mathfrak{h}_{l m}(\vec{k}, t) .
\end{gathered}
$$

If one fixes the (TT) gauge, only the (TT) components of $h_{i j}$ survive and it results

$$
h_{i j}^{(T T)}(\vec{x}, t)=\int \frac{d^{3} k}{(2 \pi)^{3 / 2}}\left(\frac{\hbar}{2 \omega(\vec{k})}\right)^{1 / 2}\left(\mathfrak{h}_{i j}^{(T T)}(\vec{k}, t) \exp (i \vec{k} \cdot \vec{x})+\mathfrak{h}_{i j}^{*(T T)}(\vec{k}, t) \exp (-i \vec{k} \cdot \vec{x})\right),
$$

and

$$
\begin{gathered}
\pi_{i j}^{(T T)}(\vec{x}, t)=\frac{1}{2} \dot{h}_{i j}^{(T T)}(\vec{x}, t) \\
=\int \frac{d^{3} k}{(2 \pi)^{3 / 2}}\left(\frac{i}{2}\right)\left(\frac{\hbar \omega(\vec{k})}{2}\right)^{1 / 2}\left(-\mathfrak{h}_{i j}^{(T T)}(\vec{k}, t) \exp (i \vec{k} \cdot \vec{x})+\mathfrak{h}_{i j}^{*(T T)}(\vec{k}, t) \exp (-i \vec{k} \cdot \vec{x})\right) .
\end{gathered}
$$

In this (TT)-gauge the Poisson brackets (6) should be severely modified since they must satisfy the Gauss' law (13). Then

$$
\left\{h_{i j}^{(T T)}(\vec{x}), \pi^{k l(T T)}\left(\vec{x}^{\prime}\right)\right\}_{P B}=\delta_{i j}^{k l(T T)} \delta\left(\vec{x}-\vec{x}^{\prime}\right),
$$

where $\delta_{i j}{ }^{k l(T T)}$ is the transverse-traceless Dirac function and it is given by

$\delta_{i j}{ }^{k l(T T)}=\frac{1}{2}\left[\left(\delta_{i}^{k}-\frac{\partial^{k} \partial_{i}}{\nabla^{2}}\right)\left(\delta_{j}^{l}-\frac{\partial^{l} \partial_{j}}{\nabla^{2}}\right)+\left(\delta_{j}^{k}-\frac{\partial^{k} \partial_{j}}{\nabla^{2}}\right)\left(\delta_{i}^{l}-\frac{\partial^{l} \partial_{i}}{\nabla^{2}}\right)-\left(\delta_{i j}-\frac{\partial_{i} \partial_{j}}{\nabla^{2}}\right)\left(\delta^{k l}-\frac{\partial^{k} \partial^{l}}{\nabla^{2}}\right)\right]$.

Then working in the temporal gauge the field $h_{i j}(\vec{x}, t)$ can be written as

$$
\begin{gathered}
h_{i j}(\vec{x}, t)=\int \frac{d^{3} k}{(2 \pi)^{3 / 2}}\left\{\varepsilon_{i j}^{a b}\left(\mathfrak{h}_{a b}^{(T T)}(\vec{k}, t) \exp (i \vec{k} \cdot \vec{x})+\mathfrak{h}_{a b}^{*(T T)}(\vec{k}, t) \exp (-i \vec{k} \cdot \vec{x})\right)\right. \\
\left.+\left(\mathfrak{h}_{i j}^{(T)}(\vec{k}, t)+\mathfrak{h}_{i j}^{*(T)}(-\vec{k}, t)+\mathfrak{h}_{i j}^{(L)}(\vec{k}, t)+\mathfrak{h}_{i j}^{*(L)}(-\vec{k}, t)\right) \exp (i \vec{k} \cdot \vec{x})\right\},
\end{gathered}
$$

where $\varepsilon_{i j}^{a b}=e_{i}^{a}(\vec{k}) e_{j}^{b}(\vec{k})$ is symmetric and traceless in $a$ and $b$ with $a, b=1,2$. Here $e_{i}^{a}(\vec{k}) \sqrt[3]{3}$ are the two well known polarization vectors expanded by the (TT)-part. These vectors are orthogonal

$$
e_{i}^{a}(\vec{k}) e_{i}^{b}(\vec{k})=\delta_{a b}, \quad k_{i} e_{i}^{a}(k)=0 .
$$

Now the Hamiltonian (4) in terms of the decomposition (11) reads

$$
H=\int d^{3} x\left(\pi_{i j}^{(T T)} \pi_{i j}^{(T T)}+\frac{1}{4} h_{k l, m}^{(T T)} h_{k l, m}^{(T T)}\right)+\int d^{3} x \mathcal{H}_{L G},
$$

\footnotetext{
${ }^{3}$ The polarization vectors $e_{i}^{a}(\vec{k})$ correspond to $\mathbf{e}_{+}$and $\mathbf{e}_{\times}$for $a=1$ and $a=2$ respectively.
} 
where

$$
\begin{aligned}
& \int d^{3} x \mathcal{H}_{L G}=\int d^{3} k \frac{\hbar}{8 \omega(\vec{k})}\left\{k_{k} \frac{\omega}{2} \mathcal{B}_{m m}^{*}(\vec{k}, t) \mathcal{A}_{0 k}(\vec{k}, t)+k_{m} k_{k} \mathcal{A}_{0 k}(\vec{k}, t) \mathcal{A}_{0 m}^{*}(\vec{k}, t)\right. \\
& \left.-\frac{1}{2}\left[\frac{\omega}{2}\left(\mathcal{C}_{m m}(\vec{k}, t) \mathcal{B}_{n n}^{*}(\vec{k}, t)+\mathcal{C}_{m m}^{*}(\vec{k}, t) \mathcal{B}_{n n}(\vec{k}, t)\right)+k_{n}\left(\mathcal{C}_{m m}(\vec{k}, t) \mathcal{A}_{0 n}^{*}(\vec{k}, t)+\mathcal{C}_{m m}^{*}(\vec{k}, t) \mathcal{A}_{0 n}(\vec{k}, t)\right)\right]\right\} \\
& +\int d^{3} k \frac{\hbar}{4 \omega(\vec{k})}\left\{\frac{k_{k} k_{l}}{4} \mathcal{A}_{0 k}^{*}(\vec{k}, t) \mathcal{A}_{0 l}(\vec{k}, t)+|\vec{k}|^{2} \mathcal{A}_{00}^{*}(\vec{k}, t) \mathcal{A}_{i i}(\vec{k}, t)-\frac{1}{2}|\vec{k}|^{2} \mathcal{A}_{i i}^{*}(\vec{k}, t) \mathcal{A}_{j j}(\vec{k}, t)\right. \\
& \left.-\mathcal{A}_{0 k}^{*}(\vec{k}, t) \mathcal{A}_{0 k}(\vec{k}, t)-k_{m} k_{n}\left(\mathcal{A}_{m l}^{*}(\vec{k}, t) \mathcal{A}_{n l}(\vec{k}, t)+\mathcal{A}_{00}^{*}(\vec{k}, t) \mathcal{A}_{m n}(\vec{k}, t)-\mathcal{A}_{m n}^{*}(\vec{k}, t) \mathcal{A}_{i i}(\vec{k}, t)\right)\right\} .
\end{aligned}
$$

Here the coefficients can be expressed by

$$
\begin{aligned}
& \mathcal{A}_{00}(\vec{k}, t) \equiv \mathfrak{h}_{00}(\vec{k}, t)+\mathfrak{h}_{00}^{*}(-\vec{k}, t), \quad \mathcal{A}_{i i}(\vec{k}, t) \equiv \mathfrak{h}_{i i}(\vec{k}, t)+\mathfrak{h}_{i i}^{*}(-\vec{k}, t), \\
& \mathcal{A}_{0 k}(\vec{k}, t) \equiv \mathfrak{h}_{0 k}(\vec{k}, t)+\mathfrak{h}_{0 k}^{*}(-\vec{k}, t), \quad \mathcal{A}_{m n}(\vec{k}, t) \equiv \mathfrak{h}_{m n}(\vec{k}, t)+\mathfrak{h}_{m n}^{*}(-\vec{k}, t), \\
& \mathcal{B}_{m m}(\vec{k}, t) \equiv \mathfrak{h}_{m m}(\vec{k}, t)-\mathfrak{h}_{m m}^{*}(-\vec{k}, t), \quad \mathcal{C}_{m m}(\vec{k}, t) \equiv \frac{\omega}{2} \mathfrak{h}_{m m}(\vec{k}, t)+k_{m} \mathfrak{h}_{0 m}(\vec{k}, t)
\end{aligned}
$$

It is easy to see that imposing the (TT)-gauge all the coefficients in (24) and consequently only the first term remains in (23) leaving only the (TT)-contribution.

Employing (11) we have that the 3 components of $\mathfrak{h}_{i j}(\vec{k}, t)$ can be written as

$$
\begin{gathered}
\mathfrak{h}_{j k}^{(T T)}(\vec{k}, t)=\mathfrak{h}_{j k}(\vec{k}, t)-\frac{k_{m} k_{k}}{|\vec{k}|^{2}} \mathfrak{h}_{j m}(\vec{k}, t)-\frac{k_{j} k_{l}}{|\vec{k}|^{2}} \mathfrak{h}_{l k}(\vec{k}, t)+\frac{1}{2} \frac{k_{j} k_{l} k_{m} k_{k}}{|\vec{k}|^{4}} \mathfrak{h}_{l m}(\vec{k}, t) \\
-\frac{1}{2} \mathfrak{h}_{m m}(\vec{k}, t) \delta_{j k}+\frac{1}{2} \delta_{j k} \frac{k_{l} k_{m}}{|\vec{k}|^{2}} \mathfrak{h}_{l m}(\vec{k}, t)+\frac{1}{2} \mathfrak{h}_{m m}(\vec{k}, t) \frac{k_{j} k_{k}}{|\vec{k}|^{2}} \\
\mathfrak{h}_{j k}^{(T)}(\vec{k}, t)=\frac{1}{2}\left(\mathfrak{h}_{m m}(\vec{k}, t) \delta_{j k}-\delta_{j k} \frac{k_{l} k_{m}}{|\vec{k}|^{2}} \mathfrak{h}_{l m}(\vec{k}, t)-\mathfrak{h}_{m m}(\vec{k}, t) \frac{k_{j} k_{k}}{|\vec{k}|^{2}}+\frac{k_{j} k_{l} k_{m} k_{k}}{|\vec{k}|^{4}} \mathfrak{h}_{l m}(\vec{k}, t)\right) \\
\mathfrak{h}_{j k}^{(L)}(\vec{k}, t)=\frac{k_{m} k_{k}}{|\vec{k}|^{2}} \mathfrak{h}_{j m}(\vec{k}, t)+\frac{k_{j} k_{l}}{|\vec{k}|^{2}} \mathfrak{h}_{l k}(\vec{k}, t)-\frac{k_{j} k_{l} k_{m} k_{k}}{|\vec{k}|^{4}} \mathfrak{h}_{l m}(\vec{k}, t) .
\end{gathered}
$$

Then under an infinitesimal diffeomorphism we have

$$
\mathfrak{h}_{i j}(\vec{k}, t) \rightarrow \mathfrak{h}_{i j}^{\prime}(\vec{k}, t)=\mathfrak{h}_{i j}(\vec{k}, t)+\xi_{i, j}+\xi_{j, i}
$$

Therefore each part transforms in the following way

$$
\begin{gathered}
\mathfrak{h}_{j k}^{\prime(T T)}(\vec{k}, t)=\mathfrak{h}_{j k}^{(T T)}(\vec{k}, t)+i\left(\delta_{j k}-\frac{k_{k} k_{j}}{|\vec{k}|^{2}}\right) k_{m} \xi_{m}, \\
\mathfrak{h}_{j k}^{(T)}(\vec{k}, t)=\mathfrak{h}_{j k}^{(T)}(\vec{k}, t)-i\left(\delta_{j k}-\frac{k_{k} k_{j}}{|\vec{k}|^{2}}\right) k_{m} \xi_{m}, \\
\mathfrak{h}_{j k}^{\prime(L)}(\vec{k}, t)=\mathfrak{h}_{j k}^{(L)}(\vec{k}, t)+\xi_{j, k}+\xi_{k, j} .
\end{gathered}
$$


Thus it is evident that the sum

$$
\mathfrak{h}^{\prime(T T)}(\vec{k}, t)+\mathfrak{h}_{j k}^{\prime(T)}(\vec{k}, t)=\mathfrak{h}_{j k}^{(T T)}(\vec{k}, t)+\mathfrak{h}_{j k}^{(T)}(\vec{k}, t)
$$

is invariant under these gauge transformations given by an infinitesimal diffeomorphism. The longitudinal $(L)$-part produces only an additional longitudinal term in $\mathfrak{h}_{i j}$ of the form

$$
\int d^{3} k\left\{i k_{j}\left(c_{k}(\vec{k}, t)+c_{k}^{*}(-\vec{k}, t)\right)+i k_{k}\left(c_{j}(\vec{k}, t)+c_{j}^{*}(-\vec{k}, t)\right)\right\} e^{i \vec{k} \cdot \vec{x}} .
$$

Thus the gauge transformation does not change $\mathfrak{p}^{i j}(\vec{k}, t), H$ and any other $(T T)$ observable.

\section{Canonical coordinates}

Similarly to other cases dealing with fields one can introduce the canonical coordinates and their conjugate momenta $\left(Q_{i j}(\vec{k}), P^{i j}(\vec{k})\right)$. Their relation with the $\mathfrak{h}$ and $\mathfrak{h}^{*}$ variables is given as usual

$$
Q_{i j}(\vec{k}):=\sqrt{\frac{\hbar}{2 \omega(\vec{k})}}\left(\mathfrak{h}_{i j}^{*}(\vec{k})+\mathfrak{h}_{i j}(\vec{k})\right), \quad P^{i j}(\vec{k}):=i \sqrt{\frac{\hbar \omega(\vec{k})}{2}}\left(\mathfrak{h}_{i j}^{*}(\vec{k})-\mathfrak{h}_{i j}(\vec{k})\right),
$$

or

$$
\mathfrak{h}_{i j}(\vec{k}):=\sqrt{\frac{\omega(\vec{k})}{2 \hbar}}\left(Q_{i j}(\vec{k})+\frac{i}{\omega(\vec{k})} P_{i j}(\vec{k})\right), \quad \mathfrak{h}_{i j}^{*}(\vec{k}):=\sqrt{\frac{\omega(\vec{k})}{2 \hbar}}\left(Q_{i j}(\vec{k})-\frac{i}{\omega(\vec{k})} P_{i j}(\vec{k})\right) .
$$

The Poisson brackets are now

$$
\begin{gathered}
\left\{Q_{i j}(\vec{k}, t), P^{k l}\left(\vec{k}^{\prime}, t\right)\right\}_{P B}=\delta_{i j}^{k l} \delta\left(\vec{k}-\vec{k}^{\prime}\right), \\
\left\{Q_{i j}(\vec{k}, t), Q_{k l}\left(\vec{k}^{\prime}, t\right)\right\}_{P B}=0=\left\{P^{i j}(\vec{k}, t), P^{k l}\left(\vec{k}^{\prime}, t\right)\right\}_{P B} .
\end{gathered}
$$

All quantities previously discussed in preceding subsections can be written in terms of $(Q, P)$ coordinates. For instance, the field $h_{i j}$ is given by

$$
h_{i j}(\vec{x}, t)=\int \frac{d^{3} k}{(2 \pi)^{3 / 2}}\left(Q_{i j}(\vec{k}) \cos (\vec{k} \cdot \vec{x}-\omega(\vec{k}) t)-\frac{P^{i j}(\vec{k})}{\omega(\vec{k})} \sin (\vec{k} \cdot \vec{x}-\omega(\vec{k}) t)\right) .
$$

The momentum is given by

$$
\pi^{i j}(\vec{x}, t)=\int \frac{d^{3} k}{(2 \pi)^{3 / 2}}\left(\omega(\vec{k}) Q_{i j}(\vec{k}) \sin (\vec{k} \cdot \vec{x}-\omega(\vec{k}) t)+P_{i j}(\vec{k}) \cos (\vec{k} \cdot \vec{x}-\omega(\vec{k}) t)\right) .
$$

These coordinates also admit a decomposition in terms of the $(T T),(T)$ and $(L)$ parts

$$
\begin{aligned}
Q_{i j}(\vec{k}) & =Q_{i j}^{(T T)}(\vec{k})+Q_{i j}^{(T)}(\vec{k})+Q_{i j}^{(L)}(\vec{k}), \\
P_{i j}(\vec{k}) & =P_{i j}^{(T T)}(\vec{k})+P_{i j}^{(T)}(\vec{k})+P_{i j}^{(L)}(\vec{k}) .
\end{aligned}
$$

\footnotetext{
${ }^{4}$ In the literature about linearized gravity these coordinates are better known as $\left(Q_{i j}^{(+)}(\vec{k}), Q_{i j}^{(-)}(\vec{k})\right)$, see for instance [6].
} 


\section{Deformation Quantization of Linearized Gravita- tional Field}

Deformation quantization in the temporal gauge

We deal now with the quantization of the linearized gravitational field in terms of the Weyl-Wigner-Groenewald-Moyal (WWGM) deformation quantization formalism. This quantization is carried out in the temporal gauge thought at the end of this section will be briefly mentioned as look like the quantization in the (TT)-gauge.

Now following the results of [11] we construct first the Stratonovich-Weyl quantizer and the Moyal $\star$-product corresponding to this system. In that reference it was used the WWGM formalism in the Wheeler's superspace. In the present paper however we prefer to use equivalently the Fourier dual, thus lets consider the fields at the moment $t=0$ and employ $\mathfrak{h}_{i j}(\vec{k}, t)$ and $\mathfrak{p}^{i j}(\vec{k}, t)$ as the coordinates of the phase space $\mathcal{Z}_{L G}$.

According to the Weyl's rule if $F=F\left[\mathfrak{h}_{i j}, \mathfrak{p}^{i j}\right]$ denotes a functional on the linearized gravitational field phase space $\mathcal{Z}_{L G}$ then we can assign to the functional $F$ the following operator $\widehat{F}$

$$
\widehat{F}=\mathcal{W}\left(F\left[\mathfrak{h}_{i j}(\vec{k}), \mathfrak{p}^{i j}(\vec{k})\right]\right):=\int \mathcal{D}\left(\frac{\mathfrak{p}^{i j}(\vec{k})}{2 \pi \hbar}\right) \mathcal{D} \mathfrak{h}_{i j}(\vec{k}) F\left[\mathfrak{h}_{i j}(\vec{k}), \mathfrak{p}^{i j}(\vec{k})\right] \widehat{\Omega}\left[\mathfrak{h}_{i j}(\vec{k}), \mathfrak{p}^{i j}(\vec{k})\right]
$$

where $\mathcal{W}$ is the Weyl correspondence map (or Weyl's image) which is an isomorphism and $\widehat{\Omega}$ stands for the operator valued distribution given by

$$
\begin{aligned}
\widehat{\Omega}\left[\mathfrak{h}_{i j}(\vec{k}), \mathfrak{p}^{i j}(\vec{k})\right]= & \int \mathcal{D}\left(\frac{\hbar \lambda^{i j}(\vec{k})}{2 \pi}\right) \mathcal{D} \mu_{i j}(\vec{k}) \exp \left\{-i \int d^{3} k\left(\lambda^{i j}(\vec{k}) \mathfrak{h}_{i j}(\vec{k})+\mu_{i j}(\vec{k}) \mathfrak{p}^{i j}(\vec{k})\right)\right\} \\
& \times \exp \left\{i \int d^{3} k\left(\lambda^{i j}(\vec{k}) \widehat{\mathfrak{h}}_{i j}(\vec{k})+\mu_{i j}(\vec{k}) \widehat{\mathfrak{p}}^{i j}(\vec{k})\right)\right\},
\end{aligned}
$$

where $\widehat{\mathfrak{h}}_{i j}(\vec{k})$ and $\widehat{\mathfrak{p}}^{i j}(\vec{k})$ are the field operators. These operators satisfy the commutation relations

$$
\left[\widehat{\mathfrak{h}}_{i j}(\vec{k}), \widehat{\mathfrak{p}}^{k l}\left(\vec{k}^{\prime}\right)\right]=i \hbar \delta_{i j}{ }^{k l} \delta\left(\vec{k}-\vec{k}^{\prime}\right),
$$

and they are defined such that acting on the corresponding states $\left|\mathfrak{h}_{i j}(\vec{k})\right\rangle$ and $\left|\mathfrak{p}^{i j}(\vec{k})\right\rangle$ fulfils the following relations

$$
\widehat{\mathfrak{h}}_{i j}(\vec{k})\left|\mathfrak{h}_{i j}(\vec{k})\right\rangle=\mathfrak{h}_{i j}(\vec{k})\left|\mathfrak{h}_{i j}(\vec{k})\right\rangle, \quad \widehat{\mathfrak{p}}^{i j}(\vec{k})\left|\mathfrak{p}^{i j}(\vec{k})\right\rangle=\mathfrak{p}^{i j}(\vec{k})\left|\mathfrak{p}^{i j}(\vec{k})\right\rangle
$$

and the completeness property that

$$
\begin{gathered}
\int \mathcal{D} \mathfrak{h}_{i j}(\vec{k})\left|\mathfrak{h}_{i j}(\vec{k})\right\rangle\left\langle\mathfrak{h}_{i j}(\vec{k})\right|=\widehat{1}, \\
\int \mathcal{D}\left(\frac{\mathfrak{p}^{i j}(\vec{k})}{2 \pi \hbar}\right)\left|\mathfrak{p}^{i j}(\vec{k})\right\rangle\left\langle\mathfrak{p}^{i j}(\vec{k})\right|=\widehat{1},
\end{gathered}
$$

where $\widehat{1}$ stands for the identity operator. 
These operators can be separated into three parts according to Eq. (11)

$$
\begin{array}{r}
\widehat{\mathfrak{h}}_{i j}(\vec{k})=\widehat{\mathfrak{h}}_{i j}^{(T T)}(\vec{k})+\widehat{\mathfrak{h}}_{i j}^{(T)}(\vec{k})+\widehat{\mathfrak{h}}_{i j}^{(L)}(\vec{k}), \\
\widehat{\mathfrak{p}}^{i j}(\vec{k})=\widehat{\mathfrak{p}}^{i j(T T)}(\vec{k})+\widehat{\mathfrak{p}}^{i j(T)}(\vec{k})+\widehat{\mathfrak{p}}^{i j(L)}(\vec{k}),
\end{array}
$$

such that their corresponding states can be written as

$$
\begin{array}{r}
\left|\mathfrak{h}_{i j}(\vec{k})\right\rangle=\left|\mathfrak{h}_{i j}^{(T T)}(\vec{k})\right\rangle \otimes\left|\mathfrak{h}_{i j}^{(T)}(\vec{k})\right\rangle \otimes\left|\mathfrak{h}_{i j}^{(L)}(\vec{k})\right\rangle, \\
\left|\mathfrak{p}^{i j}(\vec{k})\right\rangle=\left|\mathfrak{p}^{i j(T T)}(\vec{k})\right\rangle \otimes\left|\mathfrak{p}^{i j(T)}(\vec{k})\right\rangle \otimes\left|\mathfrak{p}^{i j(L)}(\vec{k})\right\rangle .
\end{array}
$$

The commutation relations for this operators are given by

$$
\begin{gathered}
{\left[\widehat{\mathfrak{h}}_{i j}^{(T T)}(\vec{k}), \widehat{\mathfrak{p}}^{k l(T T)}\left(\vec{k}^{\prime}\right)\right]=i \hbar \delta_{i j}{ }^{k l} \delta\left(\vec{k}-\vec{k}^{\prime}\right),} \\
{\left[\widehat{\mathfrak{h}}_{i j}^{(T)}(\vec{k}), \widehat{\mathfrak{p}}^{k l(T)}\left(\vec{k}^{\prime}\right)\right]=i \hbar \delta_{i j}{ }^{k l} \delta\left(\vec{k}-\vec{k}^{\prime}\right), \quad\left[\widehat{\mathfrak{h}}_{i j}^{(L)}(\vec{k}), \widehat{\mathfrak{p}}^{k l(L)}\left(\vec{k}^{\prime}\right)\right]=i \hbar \delta_{i j}{ }^{k l} \delta\left(\vec{k}-\vec{k}^{\prime}\right),}
\end{gathered}
$$

while all the others are equal to zero.

The operator $\widehat{\Omega}$ defined in Eq.(42) is the linearized gravitational field equivalent of the Stratonovich-Weyl quantizer employed in the deformation quantization of classical mechanics and we will proceed to call it in the same way.

\section{The Star-Product}

We can obtain now the Moyal $\star$-product for the linearized gravitational field. Let $F\left[\mathfrak{h}_{i j}(\vec{k}), \mathfrak{p}^{i j}(\vec{k})\right]$ and $G\left[\mathfrak{h}_{i j}(\vec{k}), \mathfrak{p}^{i j}(\vec{k})\right]$ be two functionals on $\mathcal{Z}_{L G}$, and let $\widehat{F}$ and $\widehat{G}$ be their corresponding operators given by the relations

$$
F\left[\mathfrak{h}_{i j}(\vec{k}), \mathfrak{p}^{i j}(\vec{k})\right]=\mathcal{W}^{-1}(\widehat{F}), \quad G\left[\mathfrak{h}_{i j}(\vec{k}), \mathfrak{p}^{i j}(\vec{k})\right]=\mathcal{W}^{-1}(\widehat{G}),
$$

where $\mathcal{W}^{-1}$ stands for the inverse Weyl correspondence.

In a similar way we can look for the functional which corresponds to the product of the operators $\widehat{F} \widehat{G}$. We will denote this functional by $(F \star G)\left[\mathfrak{h}_{i j}(\vec{k}), \mathfrak{p}^{i j}(\vec{k})\right]$ and following [11] we will also call it the Moyal $\star$-product. Then we define the star product as

$$
(F \star G)\left[\mathfrak{h}_{i j}(\vec{k}), \mathfrak{p}^{i j}(\vec{k})\right]:=\mathcal{W}^{-1}(\widehat{F} \widehat{G})=\operatorname{Tr}\left\{\widehat{\Omega}\left[\mathfrak{h}_{i j}, \mathfrak{p}^{i j}\right] \widehat{F} \widehat{G}\right\}
$$

Substituting (41) into (50) and after performing some calculations one obtain the following result

$$
(F \star G)\left[\mathfrak{h}_{i j}(\vec{k}), \mathfrak{p}^{i j}(\vec{k})\right]=F\left[\mathfrak{h}_{i j}(\vec{k}), \mathfrak{p}^{i j}(\vec{k})\right] \exp \left(\frac{i \hbar}{2} \stackrel{\leftrightarrow}{\mathcal{P}}_{L G}\right) G\left[\mathfrak{h}_{i j}(\vec{k}), \mathfrak{p}^{i j}(\vec{k})\right]
$$

where $\stackrel{\leftrightarrow}{\mathcal{P}}_{L G}$ denotes the Poisson operator for the linearized gravity and it is given by

$$
\stackrel{\leftrightarrow}{\mathcal{P}}_{L G}:=\int d^{3} k\left(\frac{\overleftarrow{\delta}}{\delta \mathfrak{h}_{i j}(\vec{k})} \frac{\vec{\delta}}{\delta \mathfrak{p}^{i j}(\vec{k})}-\frac{\overleftarrow{\delta}}{\delta \mathfrak{p}^{i j}(\vec{k})} \frac{\vec{\delta}}{\delta \mathfrak{h}_{i j}(\vec{k})}\right)
$$


This operator can be expressed in terms of the $(T T),(T)$ and $(L)$ components of $\mathfrak{h}_{i j}$ and $\mathfrak{p}^{i j}$ as follows

$$
\begin{aligned}
\overleftrightarrow{\mathcal{P}}_{L G} & =\int d^{3} k\left(\frac{\overleftarrow{\delta}}{\delta \mathfrak{h}_{i j}^{(T T)}(\vec{k})} \frac{\vec{\delta}}{\delta \mathfrak{p}^{i j(T T)}(\vec{k})}-\frac{\overleftarrow{\delta}}{\delta \mathfrak{p}^{i j(T T)}(\vec{k})} \frac{\vec{\delta}}{\delta \mathfrak{h}_{i j}^{(T T)}(\vec{k})}\right) \\
& +\int d^{3} k\left(\frac{\overleftarrow{\delta}}{\delta \mathfrak{h}_{i j}^{(T)}(\vec{k})} \frac{\vec{\delta}}{\delta \mathfrak{p}^{i j(T)}(\vec{k})}-\frac{\overleftarrow{\delta}}{\delta \mathfrak{p}^{i j(T)}(\vec{k})} \frac{\vec{\delta}}{\delta \mathfrak{h}_{i j}^{(T)}(\vec{k})}\right) \\
& +\int d^{3} k\left(\frac{\overleftarrow{\delta}}{\delta \mathfrak{h}_{i j}^{(L)}(\vec{k})} \frac{\vec{\delta}}{\delta \mathfrak{p}^{i j(L)}(\vec{k})}-\frac{\overleftarrow{\delta}}{\delta \mathfrak{p}^{i j(L)}(\vec{k})} \frac{\vec{\delta}}{\delta \mathfrak{h}_{i j}^{(L)}(\vec{k})}\right) \\
& =-\frac{i}{\hbar}\left\{\int d^{3} k\left(\frac{\overleftarrow{\delta}}{\delta \mathfrak{h}_{i j}^{(T T)}(\vec{k})} \frac{\vec{\delta}}{\delta \mathfrak{h}^{* i j(T T)}(\vec{k})}-\frac{\overleftrightarrow{\delta}}{\delta \mathfrak{h}^{* i j(T T)}(\vec{k})} \frac{\vec{\delta}}{\delta \mathfrak{h}_{i j}^{(T T)}(\vec{k})}\right)\right. \\
& +\int d^{3} k\left(\frac{\overleftrightarrow{\delta}}{\delta \mathfrak{h}_{i j}^{(T)}(\vec{k})} \frac{\vec{\delta}}{\delta \mathfrak{h}^{* i j(T)}(\vec{k})}-\frac{\overleftrightarrow{\delta}}{\delta \mathfrak{h}^{* i j(T)}(\vec{k})} \frac{\vec{\delta}}{\delta \mathfrak{h}_{i j}^{(T)}(\vec{k})}\right) \\
& \left.+\int d^{3} k\left(\frac{\overleftrightarrow{\delta}}{\delta \mathfrak{h}_{i j}^{(L)}(\vec{k})} \frac{\vec{\delta}}{\delta \mathfrak{h}^{* i j(L)}(\vec{k})}-\frac{\overleftrightarrow{\delta}}{\delta \mathfrak{h}^{* i j(L)}(\vec{k})} \frac{\vec{\delta}}{\delta \mathfrak{h}_{i j}^{(L)}(\vec{k})}\right)\right\}
\end{aligned}
$$

In terms of the canonical coordinates we have the Poisson operator can be written as

$$
\begin{aligned}
\overleftrightarrow{\mathcal{P}}_{L G} & =\int d^{3} k\left(\frac{\overleftarrow{\delta}}{\delta Q_{i j}^{(T T)}(\vec{k})} \frac{\vec{\delta}}{\delta P^{i j(T T)}(\vec{k})}-\frac{\overleftarrow{\delta}}{\delta P^{i j(T T)}(\vec{k})} \frac{\vec{\delta}}{\delta Q_{i j}^{(T T)}(\vec{k})}\right) \\
& +\int d^{3} k\left(\frac{\overleftarrow{\delta}}{\delta Q_{i j}^{(T)}(\vec{k})} \frac{\vec{\delta}}{\delta P^{i j(T)}(\vec{k})}-\frac{\overleftarrow{\delta}}{\delta P^{i j(T)}(\vec{k})} \frac{\vec{\delta}}{\delta Q_{i j}^{(T)}(\vec{k})}\right) \\
& +\int d^{3} k\left(\frac{\overleftarrow{\delta}}{\delta Q_{i j}^{(L)}(\vec{k})} \frac{\vec{\delta}}{\delta P^{i j(L)}(\vec{k})}-\frac{\overleftarrow{\delta}}{\delta P^{i j(L)}(\vec{k})} \frac{\vec{\delta}}{\delta Q_{i j}^{(L)}(\vec{k})}\right)
\end{aligned}
$$

\section{Wigner functional}

Let denote now by $\widehat{\rho}_{\text {phys }}$ the density operator of a physical quantum state of the linearized gravitational field, then the Wigner functional $\rho_{W}\left[\mathfrak{h}_{j k}, \mathfrak{h}^{*}{ }_{j k}\right]$ corresponding to the pure state can be obtained by

$$
\rho_{W}\left[\mathfrak{h}_{j k}(\vec{k}), \mathfrak{h}^{*}{ }_{j k}(\vec{k})\right]=\operatorname{Tr}\left\{\widehat{\Omega}\left[\mathfrak{h}_{j k}, \mathfrak{h}^{*}{ }_{j k}\right] \hat{\rho}_{\text {phys }}\right\} .
$$

In order to obtain the Wigner functional we need to solve the constraint (13) at the quantum level in the following form

$$
\nabla_{j} \widehat{\pi}^{i j}(\vec{x})\left|\Psi_{\text {phys }}\right\rangle=0,
$$


which is a constraint on the Hilbert space of states. In similar way as in [16] we introduce the equivalent form of (56) in the $h$-representation of the wave functional $\Psi_{0}$

$$
\nabla_{j} \pi^{i j}(\vec{x}) \Psi_{0}=-i \nabla_{j} \frac{\delta \Psi_{0}}{\delta h_{i j}(\vec{x})}=0 .
$$

From Eqs. (9) and (10) we obtain that the constraint equation yields

$$
\begin{aligned}
& \nabla_{j} \pi^{i j}(\vec{x}, t)=\int \frac{d^{3} k}{(2 \pi)^{3 / 2}}\left(\frac{\hbar}{2 \omega(\vec{k})}\right)^{1 / 2}\left\{\frac{-k_{j}}{2} \delta_{i j} k_{k}\left[\mathfrak{h}_{0 k}(\vec{k}, t)+\mathfrak{h}_{0 k}^{*}(-\vec{k}, t)\right]\right. \\
& \left.+\frac{k_{j} k_{j}}{2}\left[\mathfrak{h}_{0 i}(\vec{k}, t)+\mathfrak{h}_{0 i}^{*}(-\vec{k}, t)\right]+\frac{k_{j} k_{i}}{2}\left[\mathfrak{h}_{0 j}(\vec{k}, t)+\mathfrak{h}_{0 j}^{*}(-\vec{k}, t)\right]\right\} e^{i \vec{k} \cdot \vec{x}}=0 .
\end{aligned}
$$

The last equation is fulfilled if and only if the following condition holds

$$
\mathfrak{p}^{i j(L)}(\vec{k}, t)+\mathfrak{p}^{* i j(L)}(-\vec{k}, t)=0 .
$$

Or equivalently

$$
\mathfrak{h}_{0 k}^{(L)}(\vec{k}, t)+\mathfrak{h}_{0 k}^{*(L)}(-\vec{k}, t)=0 .
$$

In this way at the quantum level the constriction equation in terms of operators on the Hilbert space reads

$$
\left[\widehat{\mathfrak{h}}_{0 k}(\vec{k})+\widehat{\mathfrak{h}}_{0 k}^{*}(-\vec{k})\right]\left|\Psi_{\text {phys }}\right\rangle=0
$$

Using now the deformation quantization approach the previous equation can be written as

$$
\left[\mathfrak{h}_{0 k}(\vec{k})+\mathfrak{h}_{0 k}^{*}(-\vec{k})\right] \star \rho_{W}^{(L)}=0,
$$

which leads to

$$
\left[\mathfrak{h}_{0 k}(\vec{k})+\mathfrak{h}_{0 k}^{*}(-\vec{k})\right] \rho_{W}^{(L)}+\frac{1}{2}\left(\frac{\delta \rho_{W}^{(L)}}{\delta \mathfrak{h}_{0 k}^{*}(\vec{k})}-\frac{\delta \rho_{W}^{(L)}}{\delta \mathfrak{h}_{0 k}(-\vec{k})}\right)=0
$$

Thus this equation will be satisfied if and only if the following couple of equations is fulfilled

$$
\left[\mathfrak{h}_{0 k}(\vec{k})+\mathfrak{h}_{0 k}^{*}(-\vec{k})\right] \rho_{W}^{(L)}=0
$$

and

$$
\frac{\delta \rho_{W}^{(L)}}{\delta \mathfrak{h}_{0 k}^{*}(\vec{k})}-\frac{\delta \rho_{W}^{(L)}}{\delta \mathfrak{h}_{0 k}(-\vec{k})}=0
$$

A solution for $\rho_{W}^{(L)}$ is then given by

$$
\begin{aligned}
\rho_{W}^{(L)} & \sim \delta\left(\mathfrak{p}^{i j(L)}(\vec{k})+\mathfrak{p}^{* i j(L)}(-\vec{k})\right) \\
& \sim \delta\left(\mathfrak{h}_{0 k}(\vec{k})+\mathfrak{h}_{0 k}^{*}(-\vec{k})\right) .
\end{aligned}
$$

In terms of the $(Q, P)$-variables, the longitudinal Wigner functional is

$$
\rho_{W}^{(L)} \sim \delta\left(Q_{0 k}^{(L)}(\vec{k})+Q_{0 k}^{(L)}(-\vec{k})\right) \cdot \delta\left(P_{0 k}^{(L)}(\vec{k})-P_{0 k}^{(L)}(-\vec{k})\right) .
$$


The ground state is defined by the following equation in the Hilbert space

$$
\widehat{\mathfrak{h}}_{i j}^{(T T)}(\vec{k}, t)\left|\Psi_{\text {phys }}\right\rangle=0,
$$

which in terms of the $\star$-product reads

$$
\mathfrak{h}_{i j}^{(T T)}(\vec{k}) \star \rho_{W_{0}}^{(T T)}=0
$$

With the aid of expression (53) we get

$$
\mathfrak{h}_{i j}^{(T T)}(\vec{k}) \rho_{W_{0}}^{(T T)}+\frac{1}{2} \frac{\delta \rho_{W_{0}}^{(T T)}}{\delta \mathfrak{h}_{i j}^{(T T) *}(\vec{k})}=0 .
$$

Finally, the solution to this last equation is

$$
\rho_{W_{0}}^{(T T)}\left[\mathfrak{h}_{i j}^{(T T)}, \mathfrak{h}^{* i j(T T)}\right]=A \exp \left(-2 \int d^{3} k \mathfrak{h}^{* i j(T T)}(\vec{k}) \mathfrak{h}_{i j}^{(T T)}(\vec{k})\right),
$$

where $A$ stands by a normalization constant. It is easy to write the solution in the normal coordinates $(Q, P)$ which reads

$$
\rho_{W_{0}}^{(T T)}=A \exp \left\{-\frac{1}{\hbar} \int d^{3} k \frac{1}{\omega(\vec{k})}\left(P_{i j}^{(T T)}(\vec{k}) P^{i j(T T)}(\vec{k})+\omega^{2}(\vec{k}) Q_{i j}^{(T T)}(\vec{k}) Q^{i j(T T)}(\vec{k})\right)\right\} .
$$

Thus the general solution $\rho_{W_{0}}$ simultaneously satisfying Eqs. (62) and (69) can be written as

$$
\begin{gathered}
\rho_{W_{0}}=\rho_{W_{0}}^{(T T)} \cdot \rho_{W}^{(L)} \\
=A \exp \left(-2 \int d^{3} k \mathfrak{h}^{* i j(T T)}(\vec{k}) \mathfrak{h}_{i j}^{(T T)}(\vec{k})\right) \cdot \delta\left(\mathfrak{h}_{0 k}^{(L)}(\vec{k}, t)+\mathfrak{h}_{0 k}^{*(L)}(-\vec{k}, t)\right)
\end{gathered}
$$

or in terms of $(Q, P)$-variables we have

$$
\begin{aligned}
\rho_{W_{0}}=A \exp \{ & \left.-\frac{1}{\hbar} \int d^{3} k \frac{1}{\omega(\vec{k})}\left(P_{i j}^{(T T)}(\vec{k}) P^{i j(T T)}(\vec{k})+\omega^{2}(\vec{k}) Q_{i j}^{(T T)}(\vec{k}) Q^{i j(T T)}(\vec{k})\right)\right\} \\
& \times \delta\left(Q_{0 k}^{(L)}(\vec{k})+Q_{0 k}^{(L)}(-\vec{k})\right) \cdot \delta\left(P_{0 k}^{(L)}(\vec{k})-P_{0 k}^{(L)}(-\vec{k})\right) .
\end{aligned}
$$

\section{Normal ordering}

When we are dealing with fields at the quantum level it is usual to employ the so called normal ordering of field operators. This ordering can be extended within the deformation quantization formalism defining a proper operator acting over the functionals on the phase space. For the case of the linearized gravitational field we denote it by $\widehat{\mathcal{N}}^{(T T)}$ which acts on the $(T T)$-gauge invariant functionals on the phase space $\mathcal{Z}_{L G}$. It is written as 


$$
\begin{aligned}
\widehat{\mathcal{N}}^{(T T)} & :=\exp \left\{-\frac{1}{2} \int d^{3} k \frac{\delta^{2}}{\delta \mathfrak{h}_{i j}^{(T T)}(\vec{k}) \delta \mathfrak{h}^{* i j(T T)}(\vec{k})}\right\} \\
& =\exp \left\{-\frac{1}{2} \int d^{3} k \frac{\delta^{2}}{\delta P_{i j}^{(T T)}(\vec{k}) \delta P^{i j(T T)}(\vec{k})}+\frac{1}{\omega(\vec{k})} \frac{\delta^{2}}{\delta Q_{i j}^{(T T)}(\vec{k}) \delta Q^{i j(T T)}(\vec{k})}\right\}
\end{aligned}
$$

In this way, if $\mathcal{O}^{(T T)}\left[\mathfrak{h}_{i j}^{(T T)}(\vec{k}, t), \mathfrak{h}^{* i j(T T)}(\vec{k}, t)\right]$ represents any gauge invariant functional on $\mathcal{Z}_{L G}$ and $\widehat{\mathcal{O}}^{(T T)}$ its corresponding Weyl's image i.e. for operator $\widehat{\mathcal{O}}^{(T T)}=$ $\mathcal{W}\left(\mathcal{O}^{(T T)}\left[\mathfrak{h}_{i j}^{(T T)}(\vec{k}, t), \mathfrak{h}^{* i j(T T)}(\vec{k}, t)\right]\right)$ the normal ordering operator associated is given by

$$
: \widehat{\mathcal{O}}^{(T T)}:=\mathcal{W}\left(\widehat{\mathcal{N}}^{(T T)} \mathcal{O}\left[\mathfrak{h}_{i j}(\vec{k}, t), \mathfrak{p}^{i j}(\vec{k}, t)\right]\right) .
$$

\section{Correlation functions}

Let $\mathcal{O}$ be an invariant quantum observable and let $\widehat{\rho}_{0}$ phys be the density operator of a physical ground state. Then one quickly finds that the expected value in the ground state $|0\rangle$ reads

$$
\begin{aligned}
\langle 0|\widehat{\mathcal{O}}| 0\rangle & =\frac{\operatorname{Tr}\left\{\widehat{\mathcal{O}} \widehat{\rho}_{0}\right\}}{\operatorname{Tr}\left\{\widehat{\rho}_{0}\right\}} \\
& =\frac{\int \mathcal{D}\left(\frac{\mathfrak{p}^{i j}}{2 \pi \hbar}\right) \mathcal{D} \mathfrak{h}_{i j} \mathcal{W}^{-1}(\widehat{\mathcal{O}}) \rho_{W_{0}}\left[\mathfrak{h}_{i j}, \mathfrak{p}^{i j}\right]}{\int \mathcal{D}\left(\frac{\mathfrak{p}^{j}}{2 \pi \hbar}\right) \mathcal{D} \mathfrak{h}_{i j} \rho_{W_{0}}\left[\mathfrak{h}_{i j}, \mathfrak{p}^{i j}\right]} .
\end{aligned}
$$

The correlation function of $N$ observables $\mathcal{O}_{a}=\widehat{\mathcal{O}}\left[h_{i j}\left(\vec{x}_{a}, t_{a}\right), \pi^{i j}\left(\vec{x}_{a}, t_{a}\right)\right)$, with $a=$ $1, \cdots, N$ can be expressed in the deformation quantization approach by

$$
\begin{aligned}
\left\langle\mathcal{O}_{1} \cdots \mathcal{O}_{N}\right\rangle & =\left\langle 0\left|\widehat{\mathcal{O}}_{1} \cdots \widehat{\mathcal{O}}_{N}\right| 0\right\rangle \\
& =\frac{\int \mathcal{D}\left(\frac{\mathfrak{p}^{i j}}{2 \pi \hbar}\right) \mathcal{D} \mathfrak{h}_{i j} \mathcal{W}^{-1}\left(\widehat{\mathcal{O}}_{1}\right) \star \cdots \star \mathcal{W}^{-1}\left(\widehat{\mathcal{O}}_{N}\right) \rho_{W_{0}}\left[\mathfrak{h}_{i j}, \mathfrak{p}^{i j}\right]}{\int \mathcal{D} \mathfrak{h}_{i j} \mathcal{D}\left(\frac{\mathfrak{p}^{i j}}{2 \pi \hbar}\right) \rho_{W_{0}}\left[\mathfrak{h}_{i j}, \mathfrak{p}^{i j}\right]} .
\end{aligned}
$$

where $\mathcal{W}^{-1}\left(\widehat{\mathcal{O}}_{a}\right)=\mathcal{O}_{a}$. Of course the same is valid in the normal coordinates $(Q, P)$ system

$$
\begin{aligned}
\left\langle\mathcal{O}_{1} \cdots \mathcal{O}_{N}\right\rangle & =\left\langle 0\left|\widehat{\mathcal{O}}_{1} \cdots \widehat{\mathcal{O}}_{N}\right| 0\right\rangle \\
& =\frac{\int \mathcal{D}\left(\frac{P^{i j}}{2 \pi \hbar}\right) \mathcal{D} Q_{i j} \mathcal{O}_{1} \star \cdots \star \mathcal{O}_{N} \rho_{W_{0}}\left[Q_{i j}, P^{i j}\right]}{\int \mathcal{D}\left(\frac{P^{i j}}{2 \pi \hbar}\right) \mathcal{D} Q_{i j} \rho_{W_{0}}\left[Q_{i j}, P^{i j}\right]} .
\end{aligned}
$$

\section{Propagator}

Once the correlation functions are defined in this formalism we would like to compute the propagator of the graviton. In order to perform the functional integration 
it is convenient to use the scheme of canonical coordinates. It is easy to see that the factorization of the Wigner functional (74) and the consideration of a gauge invariant observable 5 in the following form

$$
\mathcal{O}_{i j k l}^{(T T)}=h_{i j}^{(T T)}(\vec{x}, t) \star h_{k l}^{(T T)}\left(\vec{x}^{\prime}, t^{\prime}\right)
$$

lead Eq. (79) reduces to

$$
\left\langle 0\left|\widehat{h}_{i j}^{(T T)}(\vec{x}, t) \cdot \widehat{h}_{k l}^{(T T)}\left(\vec{x}^{\prime}, t^{\prime}\right)\right| 0\right\rangle=\frac{\int \mathcal{D}\left(\frac{P^{(T T) i j}(\vec{k})}{2 \pi \hbar}\right) \mathcal{D} Q_{i j}^{(T T)}(\vec{k}) h_{i j}^{(T T)}(\vec{x}, t) \star h_{k l}^{(T T)}\left(\vec{x}^{\prime}, t^{\prime}\right) \rho_{W_{0}}^{(T T)}\left[P^{i j}, Q_{i j}\right]}{\int \mathcal{D}\left(\frac{P^{(T T) i j}(\vec{k})}{2 \pi \hbar}\right) \mathcal{D} Q_{i j}^{(T T)}(\vec{k}) \rho_{W_{0}}^{(T T)}\left[P^{i j}, Q_{i j}\right]} .
$$

Here in order to make the procedure simpler we proceed to compute (79) with only the first term in (80).

In order to compute this correlation function one has to compute first $h_{i j}^{(T T)}(\vec{x}, t) \star$ $h_{k l}^{(T T)}\left(\vec{x}^{\prime}, t^{\prime}\right)$. From (38) one can observe that it is given by

$$
\begin{gathered}
h_{i j}^{(T T)}(\vec{x}, t) \star h_{k l}^{(T T)}\left(\vec{x}^{\prime}, t^{\prime}\right)=\int \frac{d^{3} k}{(2 \pi)^{3 / 2}} \frac{d^{3} k^{\prime}}{(2 \pi)^{3 / 2}} \\
\times\left\{Q_{i j}^{(T T)}(\vec{k}) \star Q_{k l}^{(T T)}\left(\vec{k}^{\prime}\right) \cos \Theta \cos \Theta^{\prime}-Q_{i j}^{(T T)}(\vec{k}) \star \frac{P_{k l}^{(T T)}\left(\vec{k}^{\prime}\right)}{\omega\left(\vec{k}^{\prime}\right)} \cos \Theta \sin \Theta^{\prime}\right. \\
\left.-\frac{P_{i j}^{(T T)}(\vec{k})}{\omega(\vec{k})} \star Q_{k l}^{(T T)}\left(\vec{k}^{\prime}\right) \sin \Theta \cos \Theta^{\prime}+\frac{P_{i j}^{(T T)}(\vec{k})}{\omega(\vec{k})} \star \frac{P_{k l}^{(T T)}\left(\vec{k}^{\prime}\right)}{\omega\left(\vec{k}^{\prime}\right)} \sin \Theta \sin \Theta^{\prime}\right\}
\end{gathered}
$$

where $\Theta=\vec{k} \cdot \vec{x}-\omega(\vec{k}) t$ and $\Theta^{\prime}=\vec{k}^{\prime} \cdot \vec{x}^{\prime}-\omega\left(\vec{k}^{\prime}\right) t^{\prime}$. Performing the star product with the aid of (54) we observe that the first and the fourth terms have not corrections in $\hbar$, while the second and the third ones do. From the second term we have

$$
Q_{i j}^{(T T)}(\vec{k}) \star \frac{P_{k l}^{(T T)}\left(\vec{k}^{\prime}\right)}{\omega\left(\vec{k}^{\prime}\right)}=Q_{i j}^{(T T)}(\vec{k}) \frac{P_{k l}^{(T T)}\left(\vec{k}^{\prime}\right)}{\omega\left(\vec{k}^{\prime}\right)}+\frac{i \hbar}{2 \omega\left(\vec{k}^{\prime}\right)} \delta_{i k} \delta_{j l} \delta\left(\vec{k}-\vec{k}^{\prime}\right)
$$

and similarly for the third term

$$
\frac{P_{i j}^{(T T)}(\vec{k})}{\omega(\vec{k})} \star Q_{k l}^{(T T)}\left(\vec{k}^{\prime}\right)=\frac{P_{i j}^{(T T)}(\vec{k})}{\omega(\vec{k})} Q_{k l}^{(T T)}\left(\vec{k}^{\prime}\right)-\frac{i \hbar}{2 \omega(\vec{k})} \delta_{i k} \delta_{j l} \delta\left(\vec{k}-\vec{k}^{\prime}\right) .
$$

Once we substitute (83) and (84) into (82) and subsequently into (81), the first term in (83) and (84) contributes to (81) as an odd integral of the form $\int d x \exp \left(-a x^{2}\right) \cdot x$ and consequently the them vanish. Thus the only contribution to the integral (81) come from the second terms of (83) and (84). In this case we have a factor which is independent on the canonical variables $(Q, P)$ and therefore can be factor out from the

\footnotetext{
${ }^{5}$ Another important observable in the theory of gravitational waves is the Isaacson energy tensor $T_{\alpha \beta}^{G W}=\left\langle h_{i j, \alpha}^{(T T)} h_{, \beta}^{i j(T T)}\right\rangle$, which is very important to estimate the energy flux that carries the gravitational wave to be detected. The quadrupole expansion of the gravitational radiation in the (TT)-gauge also produces important observable quantities relevant in the detection of these waves.
} 
path integral giving the same gaussian integral in the numerator and the denominator and consequently they going to cancel out. Thus the second and third terms give rise to a contribution to integral (81) of the form

$$
\int \frac{d^{3} k}{(2 \pi)^{3}} \frac{i \hbar}{2 \omega(\vec{k})} \delta_{i k} \delta_{j l} \cdot \sin \left(\vec{k} \cdot\left(\vec{x}-\vec{x}^{\prime}\right)-\omega(\vec{k})\left(t-t^{\prime}\right)\right) .
$$

The star product from the first and fourth terms from (82) have no correction in $\hbar$. For the first term its is gaussian in variable $P$ and will cancel out with the denominator. The integral in $Q$ is of the form $\int d x \exp \left(-a x^{2}\right) x \cdot x$ and it is not zero. For the fourth term the $Q$-integral cancel out and the integral in $P$ is also of the form $\int d\left(\frac{p}{2 \pi \hbar}\right) \exp \left(-a p^{2}\right) p \cdot p$ and it gives a non-zero contribution. Therefore the total contribution from first and fourth terms is

$$
\int \frac{d^{3} k}{(2 \pi)^{3}} \frac{\hbar}{2 \omega(\vec{k})} \delta_{i k} \delta_{j l} \cdot \cos \left(\vec{k} \cdot\left(\vec{x}-\vec{x}^{\prime}\right)-\omega(\vec{k})\left(t-t^{\prime}\right)\right) .
$$

Gathering all, one realizes that the total contribution to the integral (81) gives finally the propagator and it is given by

$$
\left\langle 0\left|\widehat{h}_{i j}^{(T T)}(\vec{x}, t) \cdot \widehat{h}_{k l}^{(T T)}\left(\vec{x}^{\prime}, t^{\prime}\right)\right| 0\right\rangle=\int \frac{d^{3} k}{(2 \pi)^{3}} \frac{\hbar}{2 \omega(\vec{k})} \delta_{i k} \delta_{j l} \exp \left\{i\left(\vec{k} \cdot\left(\vec{x}-\vec{x}^{\prime}\right)-\omega(\vec{k})\left(t-t^{\prime}\right)\right)\right\} .
$$

This clearly a non-covariant propagator and it is the obtained here. Moreover considering the symmetry of the indices $(i j)$ and $(k l)$ and the traceless condition of $h_{i j}$ we have

$$
\begin{aligned}
\left\langle 0\left|\widehat{h}_{i j}^{(T T)}(\vec{x}, t) \cdot \widehat{h}_{k l}^{(T T)}\left(\vec{x}^{\prime}, t^{\prime}\right)\right| 0\right\rangle= & \int \frac{d^{3} k}{(2 \pi)^{3}} \frac{\hbar}{2 \omega(\vec{k})}\left(\delta_{i k} \delta_{j l}+\delta_{i l} \delta_{j k}-\delta_{i j} \delta_{k l}\right) \\
& \times \exp \left\{i\left(\vec{k} \cdot\left(\vec{x}-\vec{x}^{\prime}\right)-\omega(\vec{k})\left(t-t^{\prime}\right)\right)\right\} .
\end{aligned}
$$

Moreover from this last equation the covariant propagator can be reconstructed by taking into account the time ordering

$$
G_{i j k l}\left(x, x^{\prime}\right)=\theta\left(t-t^{\prime}\right)\left\langle 0\left|\widehat{h}_{i j}(x) \cdot \widehat{h}_{k l}\left(x^{\prime}\right)\right| 0\right\rangle+\theta\left(t^{\prime}-t\right)\left\langle 0\left|\widehat{h}_{i j}\left(x^{\prime}\right) \cdot \widehat{h}_{k l}(x)\right| 0\right\rangle,
$$

where $x$ is the four-vector $x=\left(x^{0}, \vec{x}\right), k=\left(k^{0}, \vec{k}\right)$ and $k \cdot x \equiv k_{\mu} x^{\mu}$. Thus we have

$$
\begin{aligned}
G_{i j k l}\left(x, x^{\prime}\right)= & \int \frac{d^{3} k}{(2 \pi)^{3}} \frac{\hbar}{2 \omega(\vec{k})}\left(\delta_{i k} \delta_{j l}+\delta_{i l} \delta_{j k}-\delta_{i j} \delta_{k l}\right) \\
& \times\left[\theta\left(t-t^{\prime}\right) \exp \left\{i k \cdot\left(x-x^{\prime}\right)\right\}+\theta\left(t^{\prime}-t\right) \exp \left\{-i k \cdot\left(x-x^{\prime}\right)\right\}\right],
\end{aligned}
$$

where $\theta(t)$ is the Heaviside function. It is an easy matter to see that the covariant propagator reads

$$
G_{\mu \nu \rho \sigma}\left(x, x^{\prime}\right)=\hbar \int \frac{d^{4} k}{(2 \pi)^{4}}\left(\frac{\eta_{\mu \rho} \eta_{\nu \sigma}+\eta_{\mu \sigma} \eta_{\nu \rho}-\eta_{\mu \nu} \eta_{\rho \sigma}}{k^{2}}\right) \exp \left\{i k \cdot\left(x-x^{\prime}\right)\right\} .
$$


This is precisely the graviton propagator in the literature found by the deformation quantization method.

\section{Deformation quantization in the (TT)-gauge}

Now we briefly discuss the deformation quantization of the linearized gravity in the $(T T)$-gauge (12) or (13). In this case the Poisson brackets are given by (19) in terms of the traceless transverse delta function (20). Moreover $h_{i j}(\vec{x}, t)$ and $\pi^{i j}(\vec{x}, t)$ are no longer independent variables. Thus the suitable variables are $\left(\mathfrak{h}_{i j}^{(T T)}, \mathfrak{h}^{* i j(T T)}\right)$ or $\left(Q_{i j}^{(T T)}, P^{i j(T T)}\right)$. Furthermore the Gauss law constraint (13) is automatically satisfied. Consequently it is eliminated the longitudinal parts from all formulas obtained along the paper. Then the resulting equations constitute the formulas for the deformation quantization of the linearized gravity in the (TT)-gauge.

\section{Final Remarks}

In this paper we have applied the WWGM formalism to the linearized gravitational field. The Weyl's correspondence is explicitly developed and the Stratonovich-Weyl quantizer, and the star product are constructed. These results were used to obtain the Wigner funtional $\rho_{W_{0}}$ for the ground state. It is found that this functional factorizes into two parts (see Eqs. (73) and (74)) one is the (TT)-component $\rho_{W_{0}}^{(T T)}$ and other one $\rho_{W_{0}}^{(L)}$ depending only on the longitudinal part $(L)$. Thus within the deformation quantization formalism we recover the same form for the Wigner functional of the ground state found by Kuchar [6] in the context of canonical quantization and by Hartle [7] in the path integral quantization.

Since deformation quantization is the quantum mechanics on the phase space our formulation was explicitly non-covariant, however similarly to the electromagnetic case in the temporal gauge, the formulation is shown to be covariant at the end of the quantization procedure.

In addition the normal ordering and the correlation functions were defined and the explicit computation of the propagator for the graviton was explicitly performed in Sec. 3.

Deformation quantization is a well established procedure in quantum mechanics, however in the context of field theory it still must be confirmed thought working out examples of theories of different nature. It is the goal of the present paper to continue exploring such possibilities in order to test this formalism and bring more evidence of the validity of the formalism to work in the future more complicated cases. We consider that deformation quantization possesses various advantages in order to deal with these complicated problems as to treat systems with phase spaces topologically non-trivial or with curved phase spaces. For these cases the canonical quantization could lead to the existence of non-hermitian operators which is avoided in deformation quantization as a result of the use of classical objects instead of operators. For these reasons more examples and further research is needed to develop this approximation. 


\section{Acknowledgments}

The work of H. G.-C. and F. J. T. was partially supported by SNI-México, CONACyT research grants: 103478 and 128761. In addition F. J. T. was partially supported by COFAA-IPN and by SIP-IPN grant 20110968.

\section{References}

[1] C. W. Misner, K. S. Thorne and J. A. Wheeler, San Francisco 1973, 1279p

[2] C. Will, "The Confrontation between General Relativity and Experiment," Living Rev. Relativity 9, 3 (2006) http://www.livingreviews.org/lrr-2006-3.

[3] B. Sathyaprakash and B. Schutz, "Physics, Astrophysics and Cosmology with Gravitational Waves," Living Rev. Relativity 12, 2 (2009) http://www.livingreviews.org/lrr-2009-2].

[4] R. L. Arnowitt, S. Deser and C. W. Misner, "The dynamics of general relativity," in Gravitation: An Introduction to Current Research, ed. by L. Witten (Wiley, New York, 1962), p. 227; arXiv:gr-qc/0405109.

[5] S. Deser, R. Arnowitt, Phys. Rev. 113, 745 (1959).

[6] K. Kuchar, "Ground State Functional Of The Linearized Gravitational Field," J. Math. Phys. 11 (1970) 3322.

[7] J. B. Hartle, "Ground State Wave Function Of Linearized Gravity," Phys. Rev. D 29, 2730 (1984).

[8] F. Bayen, M. Flato, C. Fronsdal, A. Lichnerowicz and D. Sternheimer, Ann. Phys. 111, 61 (1978); Ann. Phys. 111, 111 (1978).

[9] F. Antonsen, Phys. Rev. D56, 920 (1997).

[10] F. Antonsen, "Deformation Quantization of Constrained Systems", gr-qc/9710021; "Deformation Quantization of Gravity", gr-qc/9712012.

[11] R. Cordero, H. García-Compeán and F. J. Turrubiates, "Deformation quantization of cosmological models," Phys. Rev. D 83, 125030 (2011), arXiv:1102.4379 [hepth].

[12] G. Dito and D. Sternheimer, "Deformation quantization: Genesis, developments and metamorphoses," arXiv:math/0201168.

[13] C. Zachos, D. Fairlie and T. Curthright, Quantum Mechanics in Phase Space, An overwiew with selected papers, World Scientific, Singapore (2005). 
[14] B. Fedosov, J. Diff. Geom. 40, 213 (1994); Deformation Quantization and Index Theory (Akademie Verlag, Berlin, 1996).

[15] M. Kontsevich, "Deformation Quantization of Poisson Manifolds I". q-alg/9709040; Lett. Math. Phys. 48, 35 (1999).

[16] H. García-Compeán, J. F. Plebański, M. Przanowski and F. J. Turrubiates, Int. J. Mod. Phys. A 16, 2533 (2001) arXiv:hep-th/9909206.

[17] H. Quevedo and J. G. Tafoya, Gen. Rel. Grav. 37, 2083 (2005) [arXiv:gr-qc/0401088]. 